\title{
Impaired microRNA processing causes corpus luteum insufficiency and infertility in mice
}

\author{
Motoyuki Otsuka, ${ }^{1}$ Min Zheng, ${ }^{2}$ Masaaki Hayashi, ${ }^{1}$ Jing-Dwan Lee, ${ }^{1}$ Osamu Yoshino, ${ }^{3}$ \\ Shengcai Lin, ${ }^{2}$ and Jiahuai Han ${ }^{1,2}$
}

${ }^{1}$ Department of Immunology, The Scripps Research Institute, La Jolla, California, USA. ${ }^{2}$ The Key Laboratory of the Ministry of Education for Cell Biology and Tumor Cell Engineering, School of Life Sciences, Xiamen University, Xiamen, Fujian, People's Republic of China. ${ }^{3}$ Department of Obstetrics and Gynecology, Faculty of Medicine, University of Tokyo, Tokyo, Japan.

\begin{abstract}
The microRNA (miRNA) processing enzyme Dicer1 is required for zygotic and embryonic development, but the early embryonic lethality of Dicer1 null alleles in mice has limited our ability to address the role of Dicer1 in normal mouse growth and development. To address this question, we used a mouse mutant with a hypomorphic Dicer1 allele (Dicerd/d) and found that Dicer1 deficiency resulted in female infertility. This defect in female Dicer ${ }^{d / d}$ mice was caused by corpus luteum (CL) insufficiency and resulted, at least in part, from the impaired growth of new capillary vessels in the ovary. We found that the impaired CL angiogenesis in Dicerd/d mice was associated with a lack of miR17-5p and let $7 \mathrm{~b}, 2$ miRNAs that participate in angiogenesis by regulating the expression of the antiangiogenic factor tissue inhibitor of metalloproteinase 1 . Furthermore, injection of miR17-5p and let $7 \mathrm{~b}$ into the ovaries of Dicer ${ }^{d / d}$ mice partially normalized tissue inhibitor of metalloproteinase 1 expression and CL angiogenesis. Our data indicate that the development and function of the ovarian CL is a physiological process that appears to be regulated by miRNAs and requires Dicer1 function.
\end{abstract}

\section{Introduction}

Dicer is an RNase III enzyme required for processing small regulatory RNA, including siRNA and microRNA (miRNA), which respectively originate from exogenous long double-stranded RNA and endogenous single-stranded hairpin- or repeat-associated precursors (1-3). siRNA functions by means of perfect or near-perfect base pairing with mRNA targets, subsequently guiding mRNA degradation. miRNA mostly regulates gene expression by imperfect base pairing with target mRNA, subsequently guiding mRNA cleavage or translational repression (3-5). Mammals have a single Dicer gene, which presumably mediates the processing of all miRNA (3).

To determine the role of Dicer in mammals, several groups disrupted the Dicer1 gene in mice and reported that the loss of Dicer1 resulted in embryonic lethality due to either a loss of pluripotent stem cells (6) or impaired angiogenesis in the embryo (7). Tissuespecific knockouts of Dicer1 have allowed the study of Dicer1's function in selected tissues in adult mice (8-12), but the global effect of Dicer1 deficiency has not been addressed. We obtained a live Dicer1 hypomorphic expression $\left(\right.$ Dicer $\left.^{d / d}\right)$ mouse line using a gene-trap method (13), which made it possible to investigate the global effect of Dicer1 deficiency in adult mice.

Angiogenesis is the branching and sprouting of capillaries from pre-existing blood vessels and is a highly complex process in which many factors and cells are involved $(14,15)$. Although several studies have reported the involvement of miRNAs in the regulation of various molecules required for angiogenesis in vitro (16-19), the role of miRNAs in the biology of physiological postnatal angio-

Nonstandard abbreviations used: CL, corpus luteum; miRNA, microRNA; SVEC4-10, simian virus 40-transformed mouse endothelial cells; TIMP1, tissue inhibitor of metalloproteinase 1 .

Conflict of interest: The authors have declared that no conflict of interest exists. Citation for this article: J. Clin. Invest. 118:1944-1954 (2008). doi:10.1172/JCI33680. genesis in vivo is incompletely understood because of the lack of global miRNA-deficient animal models. In healthy adults, most vasculatures are quiescent, except in female reproductive organs. The functional corpus luteum (CL), which is formed from the ovulated follicle and plays a critical role in the secretion of progesterone for the maintenance of early pregnancy, requires intense angiogenesis (20-22). Although implantation failure has been proposed to explain many cases of recurrent miscarriage in humans without knowledge of the exact cause (23), luteal phase insufficiency is encountered in many of these cases (24-26). The cyclical growth of blood vessels associated with the development of the ovarian CL is essential for its function and thus for the maintenance of pregnancy $(20,21)$.

Here we report that live adult Dicer1-deficient female mice are infertile. The female infertility in this Dicer ${ }^{l / d}$ mouse line is caused by $C L$ insufficiency, which results, at least in part, from an impairment of new capillary vessel growth in the ovary. We further show that the impaired CL angiogenesis in Dicerd/d mice is partly due to a lack of miR17-5p and let7b, the 2 miRNAs that participate in the endothelial function of angiogenesis via regulation of the expression of the anti-angiogenic factor tissue inhibitor of metalloproteinase 1 (TIMP1).

\section{Results}

Female mice with hypomorphic Dicer1 expression are sterile. We established a hypomorphic Dicer1 expression mouse line using a genetrap method (13). The mouse contains a retroviral insertion in intron 24 of the Dicer 1 gene, which interferes with Dicer 1 gene expression (13). Despite the fact that the expression of Dicer1 protein is significantly reduced and miRNA production is diminished in this mouse line (13), Dicer/d mice are viable and healthy with apparently normal growth and development. However, whereas male Dicerd/d mice were fertile and produced viable offspring, 
Table 1

Infertility in female Dicerd/d mice

\begin{tabular}{|c|c|c|c|}
\hline \multicolumn{2}{|c|}{ Genotype } & \multirow[b]{2}{*}{ Mean litter size } & \multirow[b]{2}{*}{ No. of litters } \\
\hline Female & Male & & \\
\hline Dicer $^{+/+}$ & Dicer $^{+/+}$ & 8.4 & 15 \\
\hline Dicer $^{+/+}$ & Dicer+ld & 8.4 & 5 \\
\hline Dicer $^{+/+}$ & Dicerd/d & 7.5 & 5 \\
\hline Dicer+dd & Dicert/+ & 8.1 & 10 \\
\hline Dicer $^{+/ d}$ & Dicer+dd & 6.5 & 47 \\
\hline Dicert/d & Dicerd/d & 4.0 & 42 \\
\hline Dicerd/d & Dicer $^{+/+}$ & 0 & 22 \\
\hline Dicerd/d & Dicert/d $^{+/ d}$ & 0 & 6 \\
\hline Dicerd/d & Dicerd/d & 0 & 10 \\
\hline
\end{tabular}

Number of litters and litter size based on the indicated mating of wildtype, homozygous, and heterozygous male and female mice.

female Dicerd/d mice were completely sterile, regardless of whether they mated with Dicerl/d, Dicer $^{+/ d}$, or Dicer ${ }^{+/+}$males (Table 1). Reproduction in the female mice appears to be very sensitive to the level of Dicer1 activity.

Dicer1 deficiency in the ovaries is responsible for infertility in female Dicerd/d mice. To determine whether infertility in female Dicerd/d mice was due to the gonads or to systemic problems, we performed an ovary transplantation (Figure 1A). Ovaries from 4-week-old Dicer ${ }^{d / d}$ and Dicer ${ }^{+/+}$mice were exchanged into the ovarian bursas of each other and were subsequently bred to wild-type males. Five of 8 Dicer $^{d / d}$ mice transplanted with Dicer $^{+/+}$ovaries became pregnant and delivered pups (average of 3.4 pups/litter), which were all $\mathrm{Dicer}^{++}$, as expected. However, none of the Dicer ${ }^{+/+}$mice transplanted with Dicerd/d ovaries became pregnant, although they showed clear vaginal plugs. Because Dicerd/d mice can give birth to offspring when transplanted with $\mathrm{Dicer}^{+/+}$ovaries,

\section{Figure 1}

$\mathrm{CL}$ insufficiency is the cause of Dicerd/d female mouse infertility. (A) Fertility of Dicerd/d female mice transplanted with Dicer ${ }^{+/+}$ovaries. Ovaries of Dicerd/d and Dicer ${ }^{+/+}$ littermate mice were exchanged ( $n=8$ pairs), and the mice were subsequently bred to wild-type fertile males. Delivery rate and average litter size are shown. (B and $\mathbf{C})$ Ovulation and fertilization were normal in Dicer ${ }^{d / d}$ mice. (B) Representative morphologies of the 2 cell embryos collected from the oviducts of Dicerd/d mice on day 1.5 after coitus. Original magnification, $\times 100$ (top), $\times 400$ (bottom). (C) Rates of ovulation and fertilization in Dicerd/d and $\mathrm{Dicer}^{+/+}$mice were examined on day 1.5 with or without superovulation. (D) Serum progesterone levels were lower in Dicerd/d mice during pregnancy. Serum levels of progesterone in Dicerd/d mice were determined by ELISA on days $1.5,5.5$, and 7.5 of pregnancy and expressed as mean $\pm \operatorname{SD}(n=3)$. (E) The expression of genes associated with CL function was lower in Dicerd/d mice. Semiquantitative RT-PCR analyses of luteinizing hormone receptor, prolactin receptor, and cytochrome P450 family 11 subfamily a polypeptide 1 mRNA in ovaries of Dicerd/d and Dicert/+ mice were conducted on days $1.5,5.5$, and 7.5 after coitus. Results from 2 separate littermate samples are shown.

E these results indicated that the cause of the infertility was not a systemic problem (e.g., serum factors) or a defect in other parts of the reproduction system (e.g., uterus responses), but resulted because of a Dicer deficiency in the ovaries.

Luteal insufficiency is the cause of infertility in female Dicerd/d mice. Two recent studies reported that the deletion of Dicer 1 from growing oocytes prevents oocyte maturation $(27,28)$. We found that our mice with hypomorphic Dicer1 expression ovulated normally, and their ovulated oocytes were functionally normal in terms of fertilization (Figure 1, B and C). Healthy 2-cell stage embryos can be collected from Dicer ${ }^{d / d}$ mice oviducts 1.5 days after coitus (Figure $1 \mathrm{~B})$, which indicates that, unlike the oocytes, in which there is a complete deletion of Dicer1, the fertilized oocytes with hypomorphic mutant Dicer1 proceed through the first cell division successfully. In addition, the number of ovulated eggs and the rate of fertilization in Dicer ${ }^{d / d}$ mice were nearly equal to those of their wild-type littermates, with or without superovulation induced by the administration of pregnant mare serum gonadotropin plus human chorionic gonadotropin (Figure 1C).

$\mathrm{CL}$ is a transient endocrine gland and is critical to the successful maintenance of pregnancy because of its production of progesterone. Luteal phase insufficiency leads to female infertility and is the cause of such in about $9 \%$ of infertile patients $(24,25)$. The main factor associated with luteal phase insufficiency is decreased
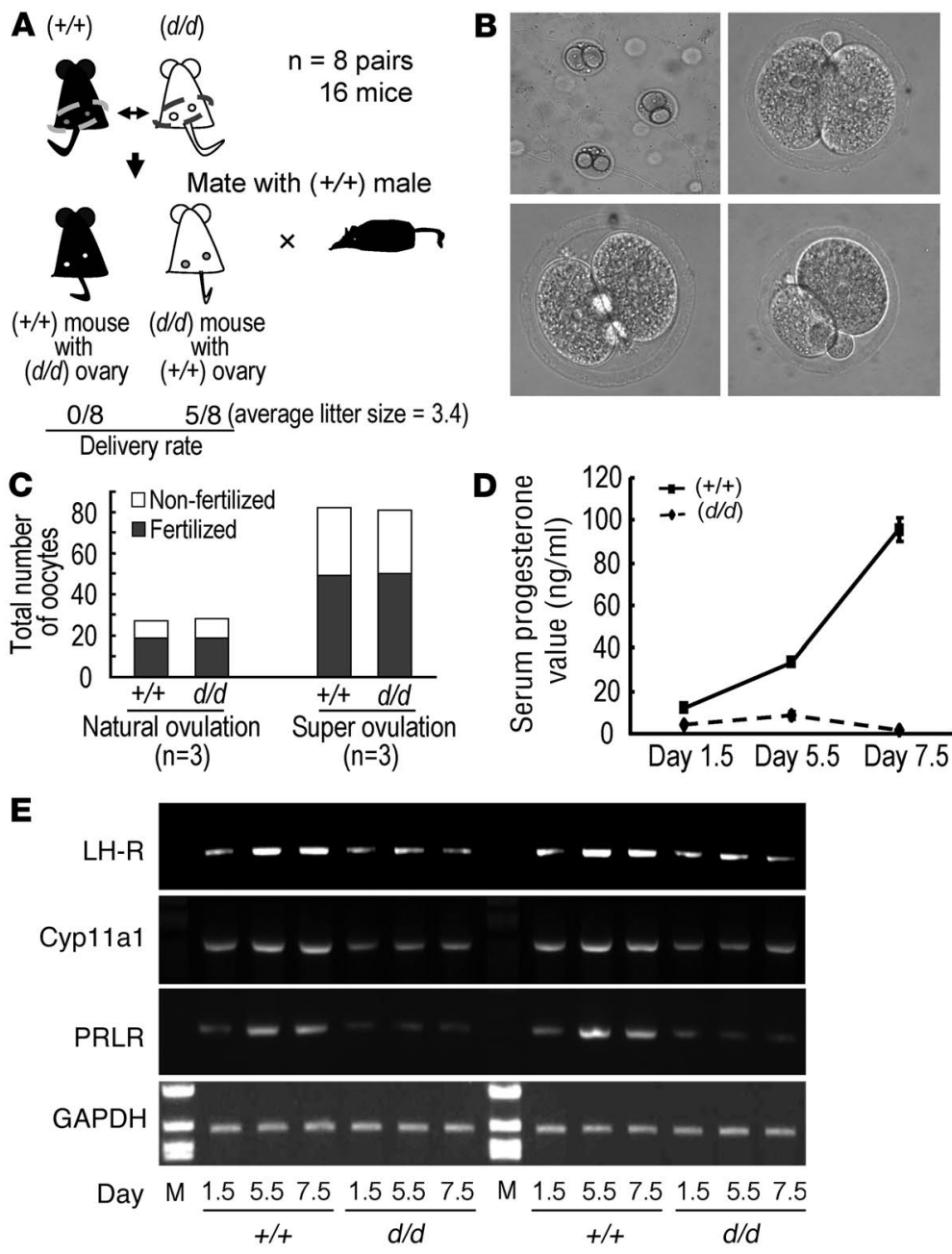

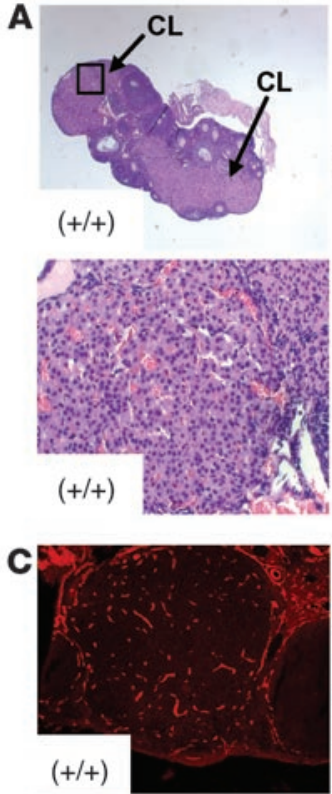

$(d / d)$
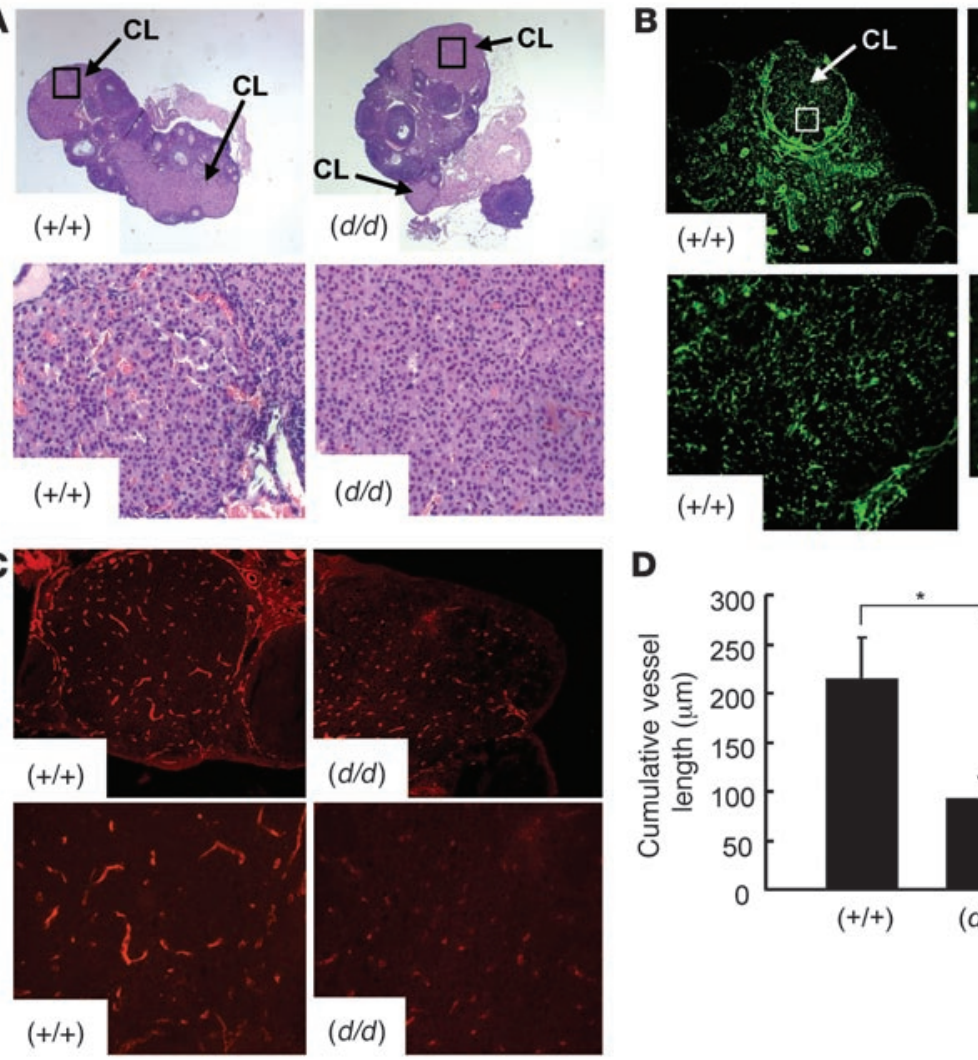

D

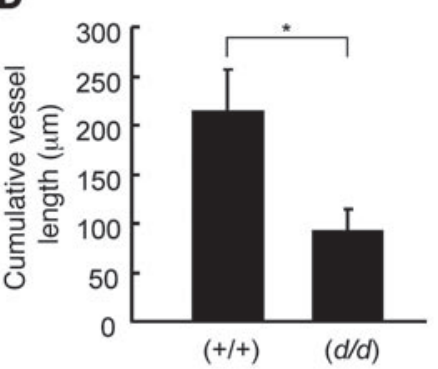

\section{Figure 2}

Impaired angiogenesis in the CLs of Dicerd/d mice. (A) Ovarian tissue sections obtained from Dicer ${ }^{+/+}$ and Dicerd/d mice on day 1.5 of pregnancy were stained with H\&E (original magnification, $\times 100$ ). CLs in $\mathrm{Dicer}^{+/+}$and Dicerd/d mice ovaries is denoted by the boxes and are shown at higher original magnification ( $\times 400$, bottom). The cells had less cytoplasm and less vascularity in the CLs of Dicerdld mice. (B) Immunofluorescence analyses of ovaries from Dicer ${ }^{d / d}$ and Dicer ${ }^{+++}$mice on day 1.5 of pregnancy show the localization of type IV collagen - a marker of the basal lamina of endothelial cells. Close examination of the vascular network in the CLs denoted by the boxes (top) is shown at higher magnification $(\times 400$; bottom). Dicer ${ }^{+/+}$CLs showed more filamentous and punctuated collagen IV staining, which indicated higher vessel densities than those in Dicerd/d mice. (C) PECAM (CD31) immunofluorescence staining of CLs in Dicerd/d and Dicer ${ }^{+/+}$mice on day 1.5 of pregnancy. The number and the length of the vessels were greater and longer in $\mathrm{Dicer}^{+/+}$CLs. Original magnification, $\times 400$. (D) Cumulative vessel length in CLs was determined as the average of the number of vessels per $100 \times 100 \mu \mathrm{m}^{2}$ multiplied by the vessel length in 3 random fields. The results of type IV collagen immunofluorescence staining from 4 different $\mathrm{Dicer}^{+/+}$and Dicerd/d pairs were used. The data are expressed as mean \pm SD. ${ }^{*} P=0.0012$.

progesterone secretion. To examine CL function, serum levels of progesterone were measured as a marker of CL function (29) 1.5, 5.5 , and 7.5 days after coitus. As shown in Figure 1D, progesterone levels were lower in Dicerd/d mice than in $\mathrm{Dicer}^{+/+}$mice at all time points (Figure 1D). The expression of genes, luteinizing hormone receptor, cytochrome $\mathrm{P} 450$ family 11 subfamily a polypeptide 1 , and prolactin receptor, which regulate the formation, function, and maintenance of the CL (30), all increased after coitus in wildtype but not in Dicer ${ }^{d / d}$ mice (Figure 1E). These results suggest that the function of CL is impaired in Dicer ${ }^{d / d}$ mice.

Angiogenesis is impaired in the CL of Dicerd/d female mice. Dicer1 is required for blood vessel formation and maintenance during embryo development (7). The formation of the CL is known to be accompanied by a dynamic angiogenesis process that involves invasion of a capillary network from the theca tissues into the granulosa cell layers (20-22). This led us to hypothesize that the
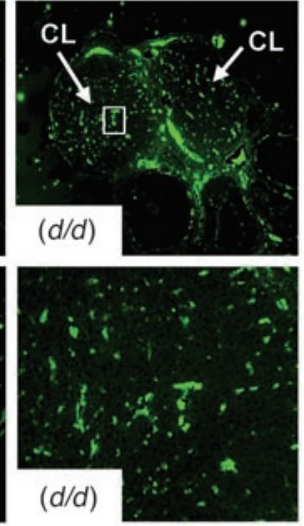

defect in ovarian function in

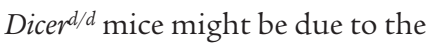
impairment of vascular development in the CL and to the subsequent insufficient functioning of the CL. To examine this possibility, Dicer ${ }^{+/+}$and Dicerd/d mice were sacrificed on day 5.5, and implantation sites in the uterus were examined. None of the

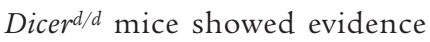
of implantation. Histological analyses of ovaries obtained on day 1.5 showed that the cells in the CLs of Dicerd/d mice had less cytoplasm and dramatically less vascularity within the CLs (Figure 2A). Ovaries of $\mathrm{Dicer}^{+/+}$and Dicer $^{d / d}$ mice on day 1.5 (a time in which an intense vascular network is established in the CL) were prepared for immunofluorescent analyses using type IV collagen as a marker of the basal lamina of endothelial cells (31) and other cell layers. As shown in Figure 2B, CLs were clearly distinguished from surrounding follicles by a complex network of collagen IV staining in both ovaries of Dicer ${ }^{+/+}$and Dicerd/d mice. However, the collagen matrix pattern is clearly different between Dicerd/d and Dicer ${ }^{+/+}$CLs. The filamentous and punctuate collagen IV staining in Dicer $^{+/+}$ CLs demonstrated higher vessel densities in Dicer $^{+/+}$CLs than in Dicerd/d CLs (Figure 2B and Supplemental Figure 1; supplemental material available online with this article; doi:10.1172/ JCI33680DS1). Staining with the endothelial marker PECAM1 (CD31) also showed a decrease in the number and length of the vessels in the CLs of Dicer ${ }^{d / d}$ mice (Figure 2C). Quantification of the cumulative vessel length in the CLs, determined by counting the number and length of the collagen IV staining, revealed fewer vessels in the CLs of Dicerd/d mice (Figure 2D). These results suggest that more arborization of the vessels occurred in $\mathrm{Dicer}^{+/+} \mathrm{CLs}$ than that in Dicer ${ }^{d / d}$ CLs. The growth of new capillary vessels in CLs is indeed impaired in Dicer $/ / d$ mice.

The antiangiogenic factor TIMP1 is upregulated in Dicerd/d ovaries. Dicer1 is unlikely to directly affect angiogenesis but is likely to regulate the expression of pro- or antiangiogenesis proteins through its processed miRNAs. Several proteins have been implicated as mediators/regulators of CL angiogenesis $(22,32,33)$. To determine whether the level of any of these proteins was changed in Dicerd/d mice, we used mouse angiogenesis antibody arrays to examine Dicer $^{+/+}$and Dicerd/d ovaries 1.5 days after coitus. The array 

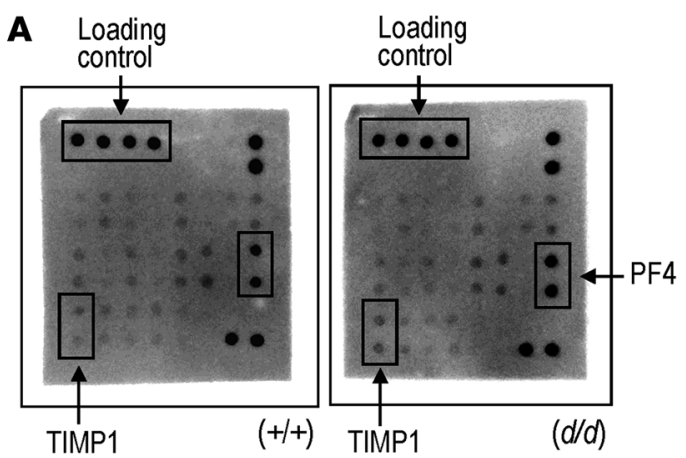

\begin{tabular}{|llllllll|}
\hline POS & POS & POS & POS & Blank & Eotaxin & FasL bFGF \\
NEG & NEG & NEG & NEG & Blank & Eotaxin & FasL bFGF \\
G-CSF & GM-CSF & IFN $\gamma$ & IGFII & IL1 $\alpha$ & IL1 $\beta$ & IL12 & IL12-p70 \\
G-CSF & GM-CSF & IFN $\gamma$ & IGFII & IL1 $\alpha$ & IL1 $\beta$ & IL12 & IL12-p70 \\
IL13 & IL6 & IL9 & Leptin & MCP1 & M-CSF & MIG & PF4 \\
IL13 & IL6 & IL9 & Leptin & MCP1 & M-CSF & MIG & PF4 \\
TIMP1 & TIMP2 & TNF $\alpha$ & TPO & VEGF & Blank & Blank & Blank \\
TIMP1 & TIMP2 & TNF $\alpha$ & TPO & VEGF & Blank & POS & POS \\
\hline
\end{tabular}

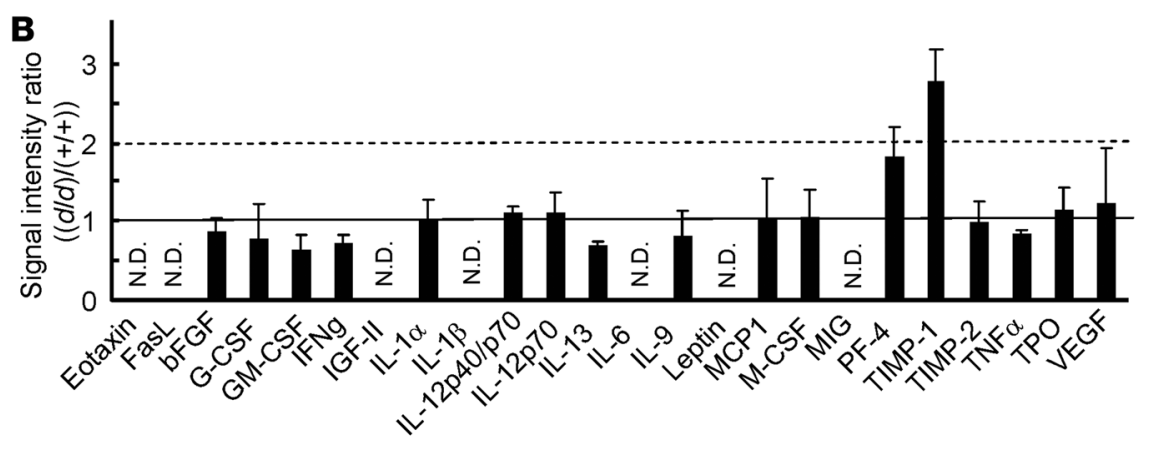

Figure 3

TIMP1 expression is elevated in Dicerd/d female mice ovaries. (A) Mouse angiogenesis-related antibody arrays hybridized with ovarian lysates from $\mathrm{Dicer}^{+/+}$and $\mathrm{Dicer}^{\mathrm{d} / \mathrm{d}}$ mice on day 1.5 after coitus. Two independent experiments using different $\mathrm{Dicer}^{+/+}$and Dicer ${ }^{d / d}$ littermate mice pairs were performed. A representative image is shown. The positions of TIMP1 are denoted by boxes and arrows. The positive loading controls are also denoted by boxes and arrows. The map of antibodies spotted on these arrays is shown below. (B) Signal intensity ratios of each gene between $\mathrm{Dicer}^{+/+}$and Dicerd/d mice ovaries obtained by protein array analyses. The normalized signal intensities of each protein from Dicerd/d mice ovaries were divided by those from Dicer $^{+/+}$mice ovaries. Four values for each gene obtained from 2 independent experimental sets were used for the analysis. The data are expressed as mean \pm SD. Genes with low signal intensity that could not be analyzed further are expressed as N.D. (not determined). The mean signal intensities of TIMP1 in Dicerd/d mice ovaries are more than 2-fold those in $\mathrm{Dice}^{+/+}$mice. (C) The distribution of $\log _{10}$ conversion values of the average of normalized signal intensities $(n=4)$ from each protein is shown as a scatter plot. The solid lines indicate the position of 2-fold changes in the intensities. The results for TIMP1 and platelet factor 4 are indicated by arrows. (D) Verification of Dicer1 protein deficiency and TIMP1 protein upregulation in Dicer ${ }^{d / d}$ mice ovaries. Dicer1 and TIMP1 protein expression in the ovaries of 2 separate Dicer ${ }^{d / d}$ and Dicer $^{+/+}$littermate mice on day 1.5 after coitus are shown. GAPDH was used as a loading control.

analysis showed that, of the 24 angiogenesis-related proteins, the expression of an antiangiogenic factor, TIMP1, was more than 2 -fold greater in Dicer ${ }^{d / d}$ mouse ovaries than in Dicer $^{+/+}$ovaries (Figure 3, A-C, and Supplemental Figure 2). The expression of platelet factor 4, another antiangiogenic factor, was also more than 1.5 -fold greater in Dicerd/d mouse ovaries. The expression of KDR (VEGFR2/Flk1) and Flt1 (VEGF1) mRNA have been reported to be elevated in Dicer-deficient whole embryos (7). However, we only detected a slight upregulation in Dicer ${ }^{d / d}$ mouse ovaries (Supplemental Figure 3). In contrast, we confirmed the increase of TIMP1 protein expression in Dicerd/d ovaries by western blot analysis (Fig- by the coexpression of miR17-5p and/or let7b, which was accompanied by decreased TIMP1 activities in reverse zymography using active MMP2-containing gels (Figure 4D). These results suggest that TIMP1 expression can be regulated by miR17-5p and let7b. We found no additive or synergistic effects of these 2 miRNAs, probably because the target sites of these 2 miRNAs mostly overlapped (Figure 4A).

$\mathrm{miR} 17-5 p$ and let $7 \mathrm{~b}$ regulate the endothelial cell angiogenic function in vitro. Because TIMP1 is known to be expressed in endothelial cells and to have a negative influence on the endothelial cell angiogenic phenotype (38-40), a simian virus 40-transformed mouse endothelial cells (SVEC4-10, or SVEC) was used to examine the relationship 
A

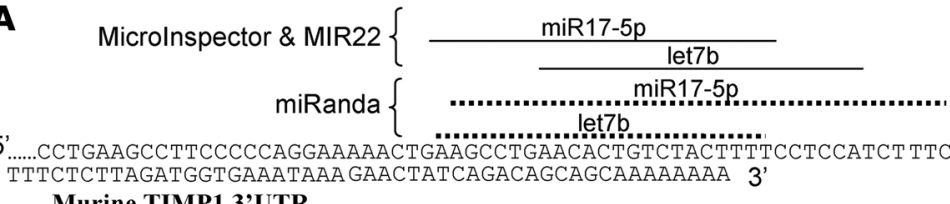
Murine TIMP1 3'UTR

Predicted by Microlnspector \& MIR22: GAAGCCTGAACACTGTC--TACTTTT 11111 111111 |11111

3' UGAUGGAC--GUGACAUUCGUGAAAC miR17-5p

Predicted by miRanda:

AGCCTGAACACTGTCTACTTTTCCTCCATCTTTC

| | | | | | | | | | | | | | | | ||

3' U-G-A-U-G-GAC-G-UG-ACA-UUCGU-GAAAC

B miR17-5p

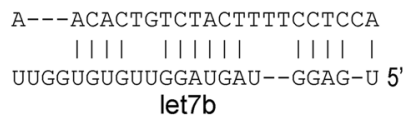

AAGCC-TG-AACACTGTCTACTTTT || || : : ||| ||| ||||||| UU-GGUGUGUUG-GAU-GAUGGAGU 5 let $7 \mathrm{~b}$

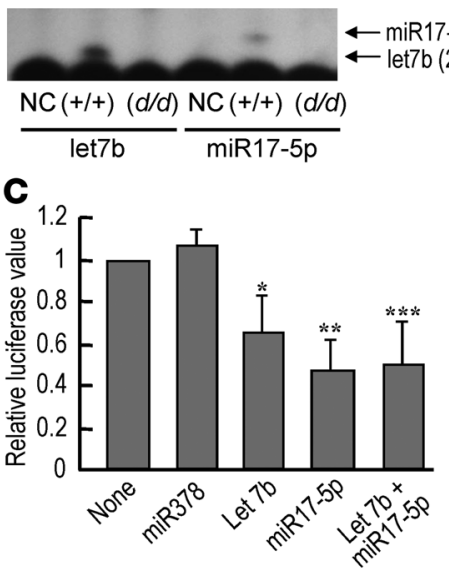

\section{Figure 4}

miR17-5p and let7b can target 3'-UTR of mTIMP1. (A) miRNA target positions in the $3^{\prime}$-UTR of MTIMP1 were identified by computational prediction methods, Microlnspector, and miRanda. The same candidate miRNAs, miR17-5p and let7b, were predicted by the different programs; however, the predicted miRNA-targeting sequences in the $3^{\prime}$-UTR of TIMP1 are not exactly the same. (B) Primer extension analyses showed the expression of the indicated miRNAs in Dicer+/+ and Dicerd/d mice ovaries. NC, negative control. (C) miR17-5p and let7b can target 3'-UTR of TIMP1. The 293T cells were transiently transfected with a reporter plasmid (pLuc-TIMP3'-UTR) with or without indicated miRNA expression plasmids, and 36 hours after transfection, a reporter assay was performed. The relative luciferase values were calculated by dividing firefly luciferase values with internal control renilla luciferase values. The value from the negative control was set at 1. Data are shown as mean \pm SD from 3 independent experiments. ${ }^{*} P=0.032$, ${ }^{* *} P=0.00082,{ }^{* * *} P=0.0035$. (D) miR17-5p and let7b downregulate TIMP1 expression and activity. The 293T cells were transiently transfected with pcDNA-TIMP1, with or without the indicated miRNA expression plasmids, and pRL-TK. TIMP1 expression was examined by western blotting 36 hours after transfection (top). Cell extracts were normalized based on the luciferase value of cotransfected $p R L-T K$ to avoid the variances of transfection efficiency. Bottom: Result of reverse zymography using the MMP2-containing gels and the concentrated culture supernatant collected from the transfected cells to quantitate the activities of TIMP1 against MMP2 activities. A representative result from 4 independent experiments is shown. Numbers below the images refer to the fold changes of the intensities. between the TIMP1-targeting miRNAs and endothelial cell angiogenic function in vitro. Because one of the most specific tests for in vitro approximation of angiogenesis is the measurement of the ability of endothelial cells to form 3-dimensional structures (tube formation) (41), we examined the tube formation by SVEC placed on Matrigel. The inhibition of miR17-5p and let7b by complementary 2'-O-methyl oligonucleotides in SVEC significantly impaired tubeforming activity (Figure 5A). In contrast, overexpression of miR17$5 \mathrm{p}$ and let7b significantly increased endothelial tube formation (Figure 5A). The statistical significance of the effect of inhibiting or increasing miR17-5p and let7b in SVEC on tube formation was determined by measuring tube length 12 and 18 hours after seeding on Matrigel (Figure 5B). The expression of miR17-5p and let7b in SVEC was confirmed by primer extension analyses (Figure $5 \mathrm{C})$. The change in tube-forming activity was not dependent on the change in cell proliferation or cell motility, because they were not affected by the transfection of miRNA inhibitors or precursors (Figure 5, D and E). In contrast, the levels of TIMP1 activity against MMP2 activity changed after transfection with miRNA inhibitors or precursors (Figure 5F). These results suggest that miR17-5p and let7b are important for the angiogenic activity of endothelial cells through the modulation of TIMP1 and MMP activities.

In vivo miRNA injection restores angiogenesis in the CL of Dicerd/d female mice. To evaluate the role of miR17-5p and let7b on CL angiogenesis in vivo, these miRNAs were injected into Dicer ${ }^{d / d}$ mouse ovaries. We tried to inject miRNAs into ovarian medulla tissues directly; however, in this case, female mice did not accept mating, typically for more than 3 weeks after treatment, probably because of the damage or stress of the ovaries associated with the procedure. In another attempt, the transfection reagent mixture was injected into the ovarian bursa, which resulted in the ovary floating in the transfection complex to be covered with ovarian bursa. To determine the transduction efficiency of this technique, fluorescencelabeled RNA oligonucleotides were used as a marker. Three days after transfection, the ovaries in the frozen section were examined under a fluorescent microscope. The labeled RNA was distributed in the whole treated ovaries, although there was an inconsistency in the transduction efficiency among different cell type components (Figure 6A). Next, miRNAs were transfected into one ovary in each Dicerd/d female mouse and, as a control, only transfection reagents were injected in the other ovary. On the next day of the treatment, the mice were housed with fertile males, and they typically mated within 5 days of treatment. The patterns of a marker of the basal lamina of endothelial cells - type IV collagen - in the CLs were found to be more filamentous and punctuated in the miRNA-introduced ovaries than were those in the control ovaries 1.5 days after coitus, and cumulative vessel length in the CLs was also recovered (Figure 6, B and C, and Supplemental Figure 4). Consistent with this, TIMP1 expression in the miRNA-injected ovaries was indeed suppressed 1.5 days after coitus (Figure 6D). The suppression of TIMP1 expression after miRNA injection was also confirmed by antibody arrays using the ovaries 1.5 days after coitus (Figure 6E and Supplemental Figure 5). Moreover, serum progesterone values in Dicerd/d mice with the exogenously introduced miRNA precursors into both ovaries were also partially recovered, at least until day 5.5 of pregnancy (Figure 7C). These 
A Negative control
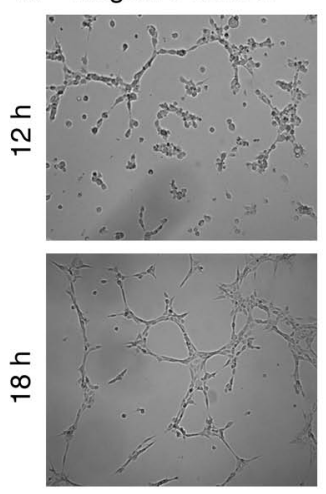

Anti-let7b + anti-miR17-5p oligos
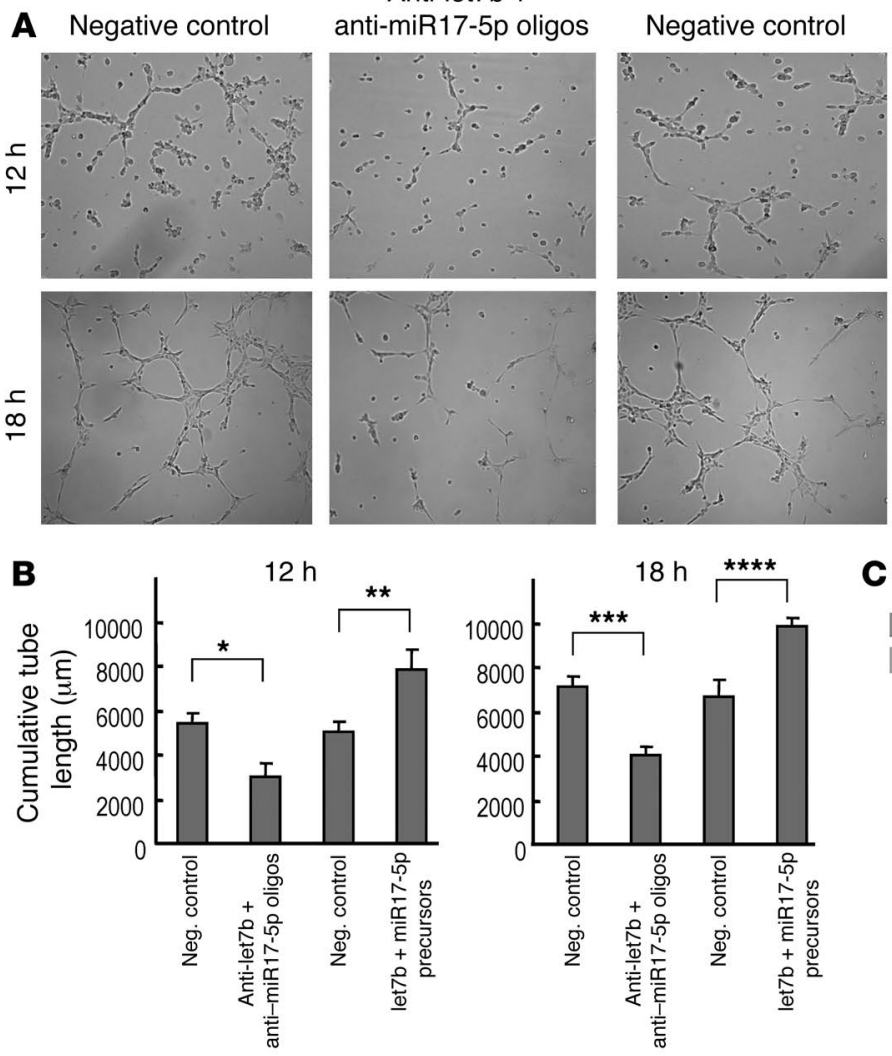

D

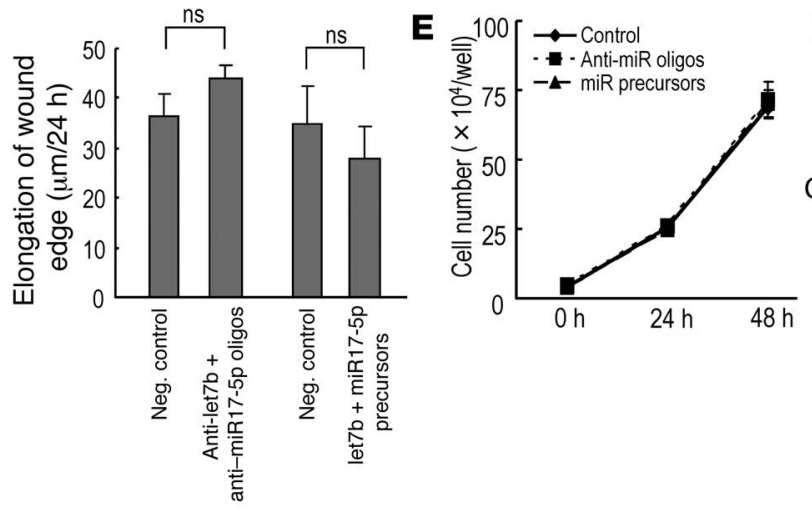

let7b + miR17-5p precursors
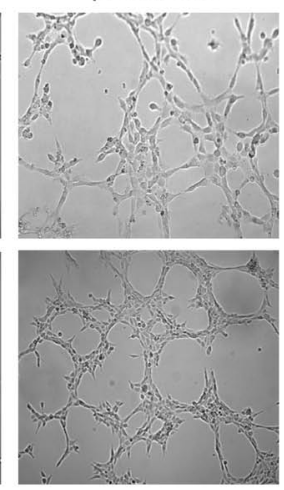

C

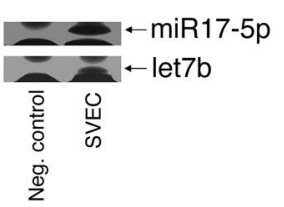

F

TIMP1 activity $\rightarrow$ $\begin{array}{lllll}\text { Fold change } & 1 & 1.79 & 1 & 0.66\end{array}$

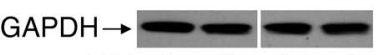

\section{Figure 5}

miR17-5p and let7b control endothelial cell angiogenic function. (A and $\mathbf{B}$ ) Indicated anti-miRNA oligonucleotides or miRNA precursors were transfected into SVEC. Thirty-six hours after transfection, cells were seeded on Matrigel. Tube length was quantified after 12 and 18 hours. Representative micrographs (A) and statistical summary (B) are shown. Data represent mean \pm SD of 3 experiments. ${ }^{*} P=0.0014,{ }^{* *} P=0.0057$, ${ }^{\star \star \star *} P=0.000055,{ }^{\star \star \star \star} P=0.00067$. (C) Primer extension analyses determined the expression of the indicated miRNAs in SVEC. (D) miR17-5p and let7b had no effect on the proliferation of SVEC. Thirty-six hours after transfection of the indicated oligos into SVEC, $5 \times 10^{4}$ cells were seeded, and the number of cells was determined by cell counting at the indicated time points. Data are presented as mean \pm SD $(n=4$ per group, $P=\mathrm{NS}$ ). (E) miR17-5p and let7b had no effect on the motility of SVEC. In vitro wound healing was quantified as the average length of the elongation of wound edges at 4 specific points over 24 hours. Data are presented as mean $\pm \mathrm{SD}(n=3$ per group, $P=\mathrm{NS})$. (F) The activities of TIMP1 against MMP2 were modulated by miRNAs. A representative result of reverse zymography is shown using the the SVEC culture supernatant after transfection with the oligos (lane 1, control antisense; lane 2, anti-miRNA17-5p and let7b; lane 3, control miRNA precursors; lane 4, miR17-5p and let7b precursors). The numbers indicate the fold changes of the band intensities. GAPDH expression did not change after transfection of oligos. The experiments were repeated 3 times with similar results. results suggest that miR17-5p and let7b can partially recover the vascularity in the CLs, likely in part through the suppression of TIMP1 expression in Dicer ${ }^{d / d}$ mouse ovaries.

In contrast, transduction of anti-miRNA inhibitors into $\mathrm{Dicer}^{+/+}$ mouse ovaries resulted in decreased vessel development in CLs (Figure 7A) with upregulation of TIMP1 expression in the ovaries (Figure 7B). Dicer ${ }^{+/+}$mice in which anti-miRNA inhibitors were exogenously introduced into both ovaries also showed a decrease in serum progesterone levels and could not maintain their pregnancy. However, this failure was not considered to be solely due to the effects of anti-miRNA inhibitors, because Dicer $^{+/+}$mice injected only with transfection reagents also showed decreased progesterone levels after day 5.5 of pregnancy and could not maintain their pregnancy (Figure 7C). The failure to maintain pregnancy may have been due to the nonspecific effects associ- ated with the injection and transfection procedure. Nonetheless, anti-miR17-5p and let7b inhibitors upregulated TIMP1 expression in the ovaries, decreased vascularity in the CLs, and decreased serum progesterone values in $\mathrm{Dicer}^{+/+}$mice during an earlier phase of their pregnancy.

Similarly, although the introduction of miR17-5p and let7b improved the vascularity of the CLs, as well as the serum progesterone levels of Dicerd/d mice, none of these mice maintained their pregnancy to a later phase. The most likely explanation was the possible requirement of many other miRNAs during the process of pregnancy, such as the participation of unidentified miRNA(s) that target some other angiogenesis-related factor(s), or the involvement of some unknown miRNAs in the development during the later phase, such as placental formation. Therefore, injection of just 2 miRNAs could not fully restore 

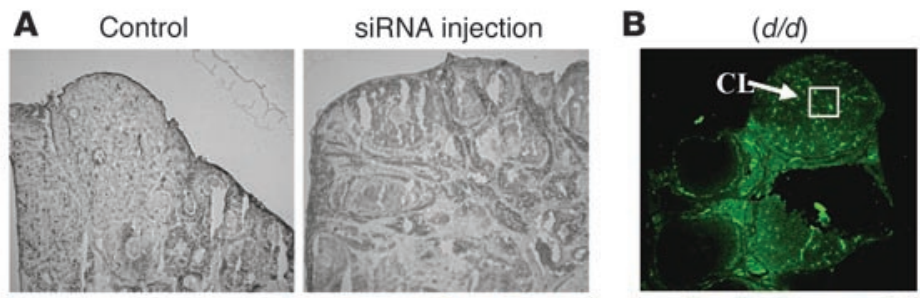

$(d / d)+$ miR precursors injection
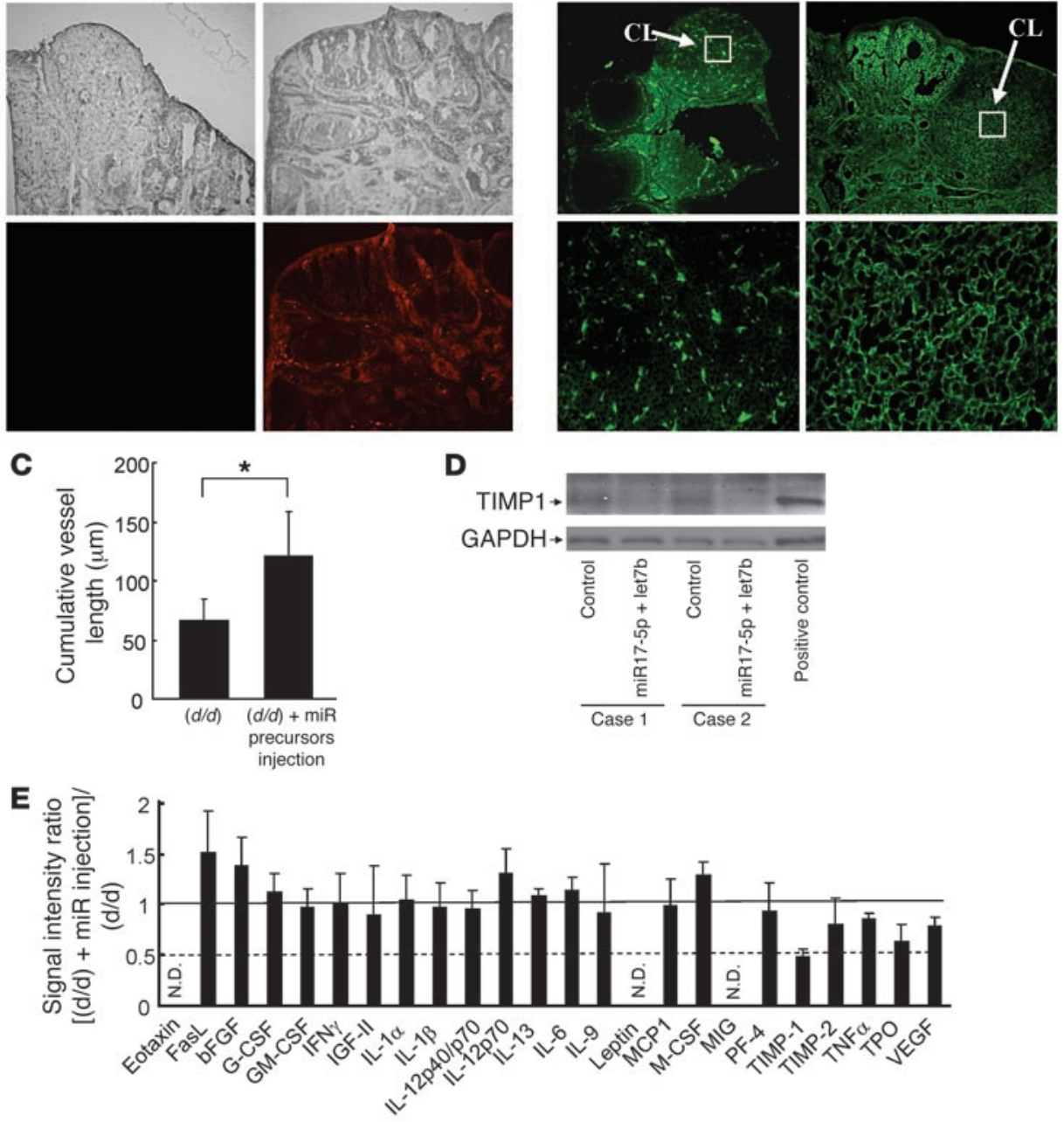

\section{Figure 6}

miR17-5p and let7b recover the vascularity in the CLs in Dicerd/d mouse ovaries in vivo. (A) Cy-3-labeled siRNA was injected into the ovarian bursa. Injection of transfection reagent alone was used as control. Top: Images in the bright field. Bottom: Same fields in the fluorescent phase. Original magnification, $\times 100$. (B) miR17-5p and let7b recover the vascularity in CLs. miRNA precursors were injected into the ovarian bursa of Dicer $^{d / d}$ mice, and ovaries were stained with anti-type IV collagen, as described in Methods. Top: original magnification, $\times 100$. Close examination of the vascular network denoted by the boxes are shown (original magnification, $\times 400$; bottom). (C) Cumulative vessel length in CLs in the miRNA-injected ovaries $[(d / d)+$ miR precursor injection] and the corresponding control ovaries $(d / d)$ was determined as described in the legend of Figure 2D. Results are from 3 different Dicerd/d mice. Data represent mean \pm SD. ${ }^{*} P=0.0031$. (D) miR17-5p and let7b suppressed the expression of TIMP1 in the ovaries in vivo. miRNA precursors were injected into the ovaries as described in Methods. TIMP1 protein expression in the ovaries of 2 separate miRNA-injected mice are shown. SVEC lysates were used as a positive control. GAPDH was used as a loading control. (E) The suppression of TIMP1 expression in the ovary was confirmed by an antibody array. Signal intensity ratios of each gene between the miRNA-introduced Dicer ${ }^{d / d}$ ovaries $\left(d / d+\right.$ miR injection) and the corresponding control Dicer $r^{d / d}$ ovaries $(d / d)$ are shown after normalization. Results are the summary of 4 values of each gene from 2 independent experiments using different mice.

all processes necessary to maintain pregnancy. Other possible explanations were as follows: the amounts of miR17-5p and let7b introduced into the ovaries of these mice were insufficient, the transfected miRNAs might not last very long in vivo, and the nonspecific physiological and/or endocrine stress directly derived from the procedure and subsequent wound healing might affect the course of pregnancy, as was observed in antimiRNA inhibitor transduced Dicer ${ }^{+/}$mice. Nevertheless, we concluded that diminished miRNA production in Dicerd/d mice impairs CL angiogenesis.

\section{Discussion}

The study described in this article indicates that the function of the ovary is tightly regulated by miRNA, because the most obvious effect of the hypomorphic Dicer1 mutation is ovary dysfunction-mediated female infertility. Mammalian reproduction is a multistep process, including folliculogenesis, oocyte maturation, ovulation, fertilization, cleavage, implantation, and maintenance of pregnancy (42-44). Although oocytes cannot mature without Dicer1 $(27,28)$, our data show that a reduction in Dicer1 expression only affected implantation and the maintenance of pregnancy. 
A

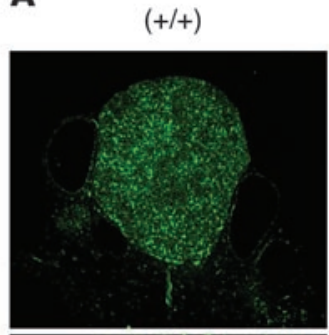

$(+/+)+$ miR inhibitors injection
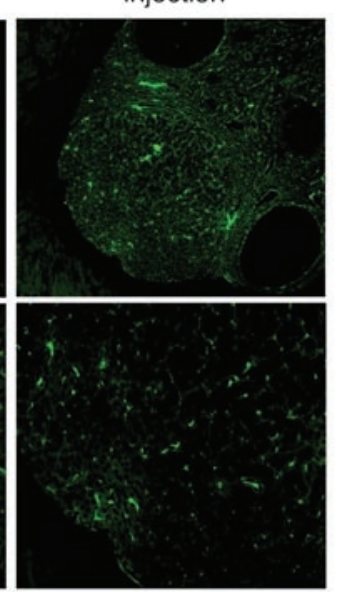

B

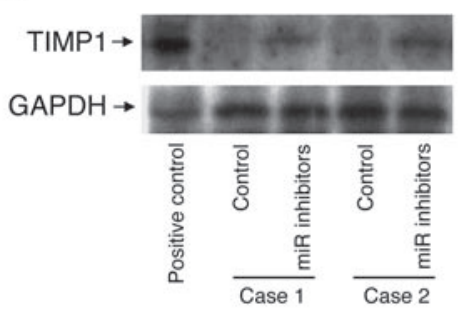

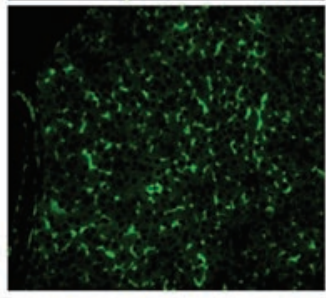
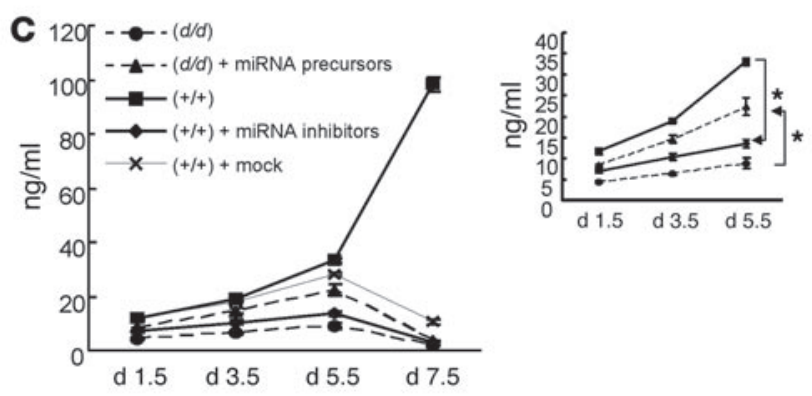

Figure 7

Anti-miR17-5p and let7b oligonucleotides impaired the vascularities in the CLs in Dicer ${ }^{+/+}$ mouse ovaries. (A) A mixture of anti-miRNA17-5p and let7b oligonucleotides and transfection reagent, or transfection reagent only, were injected into the right or left ovarian bursa of $\mathrm{Dicer}^{+/+}$mice, respectively, and the patterns of type IV collagen in the CLs were examined as described in the legend of Figure 6B. A representative result from 3 independent mice is shown. (B) Anti-miR17-5p and let7b oligonucleotides enhanced the expression of TIMP1 in the ovaries in vivo. TIMP1 protein expression in the ovaries of mice injected with 2 anti-miR17-5p plus anti-let7b oligonucleotides. SVEC lysates were used as positive control for the detection of TIMP1. GAPDH was used as a loading control. (C) Serum progesterone levels were modified in miRNA precursors or anti-miRNA inhibitor-injected mice. Dicerd/d mice injected with a mixture of miRNA17-5p and let7b precursors into both side ovaries $[(d / d)+$ miRNA precursors $]$ had greater serum progesterone levels than did Dicerd/d mice $(d / d)$ until day 5.5 of pregnancy. In contrast, Dicer ${ }^{+/+}$mice injected with a mixture of anti-miRNA17-5p and let7b inhibitors into both ovaries $\left[(+/+)+\right.$ miRNA inhibitors] had lower serum progesterone levels than did $\mathrm{Dicer}^{+/+}$ mice $(+/+)$. The serum progesterone levels of Dicer $^{+/+}$mice injected only with transfection reagents into both side ovaries are also shown $[(+/+)+$ mock]. Serum progesterone levels were determined by ELISA on days 1.5, 3.5, 5.5, and 7.5 of pregnancy and expressed as mean \pm SD ( $n=3$ in each group). A graph of results until day 5.5 is shown in the inset.

The defect in the growth of new capillary vessels in the CLs was at least part of the mechanism underlying the CL insufficiency found in Dicer ${ }^{d / d}$ mice. Although we used TIMP1 to determine the role of miR17-5p and let7b in CL development, other miRNA-regulated proteins may also participate in CL angiogenesis and, thus, other unknown miRNAs may also be important to CL functioning. Several miRNAs have been implicated in the control of various angiogenesis-related molecules and thus they may positively or negatively affect angiogenesis in a system-dependent manner (16-19). Whether miRNAs are commonly involved in angiogenesis in the $\mathrm{CL}$ and other systems requires further investigation. Whereas miR17-5p and let7b are involved, CL insufficiency in Dicerd/d mice should be the net consequence of the global downregulation of miRNAs.

It should be noted that our results do not exclude the possibility that other ovarian functions, such as folliculogenesis, oocyte maturation, ovulation, or other endocrine functions, are regulated by miRNAs. However, our hypomorphic Dicer1 mutant mice showed that the development and function of the ovarian $\mathrm{CL}$ is the physiological process that appears to be most sensitive to miRNAs' deficiency during the early phase of pregnancy.

Recent studies have shown that oocytes with Dicer1 deletion failed to progress through first cell division $(27,28)$. However, the oocytes of our Dicer1-deficient mice appeared to be normal in this process, which suggested that the reduction in Dicer1 protein in Dicer ${ }^{d / d}$ oocytes was still sufficient for oocyte maturation. Deletion of exon 1 and exon 2 of the Dicer 1 gene impaired angiogenesis during embryonic development (7), whereas hypomorphic Dicer1 expression in our Dicer/d/ mice did not affect angiogenesis during embryonic development, but did affect angiogenesis in the CL. This finding indicated that angiogenesis in different tissues has different sensitivities to Dicer1 protein levels. Because different miRNAs were identified as regulating angiogenesis in different systems (16-19), it is possible that these different sensitivities to Dicer1 protein levels are due, in part, to the different miRNAs involved in the angiogenesis within the $\mathrm{CL}$ and embryo.

Because the deletion of Dicer1 is embryonically lethal (6), live hypomorphic Dicer1 mutant mice should serve as a useful system for analyzing Dicer1's role in development- and health-related issues in adult animals. The existence of natural cases of total knockout of Dicer 1 or total knockout of Dicer 1 in certain tissues, similar to the conditional knockout mice, are unlikely because Dicer1 is critical to survival. Studying the changes in Dicer1 expression or functional deficiency that result from single nucleotide polymorphisms or hypomorphic mutations in Dicer 1 is potentially important in determining whether Dicer1 is involved in a given disease. It may be worth analyzing the expression and sequence of the Dicer 1 gene in various angiogenesis-related diseases, especially in cases of female infertility, as shown here. This study demonstrates that miRNAs can be involved in the pathogenesis of CL malfunction and may have therapeutic implications, not only for the control of fertility, but also for the treatment of ovarian disorders and other pathological conditions characterized by abnormal angiogenesis. 


\section{Methods}

Experimental animals. Dicer-deficient mice (129/Ola and C57BL/6 mixed background), obtained using a gene-trap method, were described previously (13). The mice used in this study were female offspring littermates derived from crosses between male and female $\mathrm{Dicer}^{+/ d}$ mice after more than 10 generations of sibling breeding (13). All experiments were carried out in compliance with the rules of, and were approved by, the Animal Use Committee of The Scripps Research Institute.

Timed mating experiments. For timed mating experiments, Dicer ${ }^{+/+}$and Dicer $^{d / d}$ female mice were bred with fertile wild-type males. To induce superovulation, mice were first injected (i.p.) with pregnant mare's serum gonadotropin (5 IU/mouse; Sigma-Aldrich) and then with human chorionic gonadotropin (5 IU/mouse; Sigma-Aldrich) 48 hours later. The female mice were then bred with male mice overnight. The following morning (day 0.5 after coitus), the female mice were checked for a vaginal plug. The mice were sacrificed on day 1.5. Oocytes and 2- and 4-cell stage embryos, collected by flushing the oviducts with PBS, were examined under a dissecting stereomicroscope.

Ovary transplantation. Ovary transplantation was performed as described previously (45) using 4- to 6-week-old female littermate mice to avoid immunological rejection of tissues. After anesthesia with ketamine, a dorsolateral incision about $1.0 \mathrm{~cm}$ long was made in the lumber region on each side of the midline. Through the incision, the ovaries were gently pulled outside the body. The ovarian bursa was cut to make a small incision at the opposite side of the oviduct. The ovaries were removed by cutting the ovarian stalk with scissors. After the bleeding stopped, donor ovaries were transplanted into the empty bursas of each recipient mouse. The bursas were placed back, and the reproductive tract was returned to the abdominal cavity. The skin incision was closed with sutures. Two weeks after the operation, treated mice were mated with fertile male mice. The genotype of the offspring born to the recipient mice was confirmed by PCR (13).

Histological analysis, immunohistochemistry, and immunofluorescence. Tissues were dissected out, fixed with $4 \%$ paraformaldehyde, embedded in paraffin, sectioned, and analyzed by H\&E staining. For type IV collagen immunofluorescent studies, sections were stained with primary antitype IV collagen (Abcam) and secondary Alexa Fluor 488-conjugated goat anti-rabbit IgG antibodies (Molecular Probes). For CD31/PECAM immunofluorescent studies, frozen sections were incubated with primary anti-CD31/PECAM (MEC 13.3; BD Pharmingen) and then with secondary Alexa Fluor 594-conjugated goat anti-rat IgG antibodies (Molecular Probes). Slides were mounted using VectaShield (Vector Labs). Cumulative vessel length in CLs was evaluated as the average of the number of vessels per $100 \times 100 \mu \mathrm{m}^{2}$ multiplied by the vessel length in 3 random fields at $\times 400$ fields from 4 different pairs.

Angiogenesis-related protein antibody array. Mouse Lysate Angiogenesis Antibody Array, which detects 24 different kinds of angiogenesis-related cytokines, was purchased from Chemicon. Dicer ${ }^{+/+}$and Dicer/d/ mouse ovaries, day 1.5 after coitus, were used for the screening according to the manufacturer's instructions. The data were evaluated using FluorChem8900 software (Alpha Innotech). A grid containing a series of circles corresponding to the spots of the array was laid over the image, and the circles were adjusted to each spot. The intensities within the circle were calculated, and the local background intensities around the circle (intensities in the outer torus with 1.3-fold longer diameter of the spot circle) were subtracted to reduce bias. Genes whose signal intensities were lower than 5 times their background intensities were excluded from further analyses to exclude the variability due to their low expression levels. The normalized signal intensities of each gene were determined as the intensities divided by the average intensities of positive control spots on the same array. Relative protein expression levels between different samples were determined as signal intensity ratios, obtained by dividing the normalized signal intensities of each gene from different samples. Because each gene has 2 spots on one array, 4 values for each gene derived from 2 independent experimental sets were used for one statistical analysis. A scatter plot was depicted using $\log _{10}$ conversion of the average of normalized signal intensities of each gene.

Primer extension. Primer extension was performed as described previously (5). The primer sequences of miR17-5p and let7b are ACTACCTGCACTGTAAGC and AACCACACAACCTACTAC, respectively.

Prediction of miRNA target sites. Two computational miRNA target prediction programs, MicroInspector (34) and miRanda (36), were used to predict miRNA binding sites in $3^{\prime}$-UTR mTIMP-1 sequences. MicroInspector was run with the hybridization temperature set to $37^{\circ} \mathrm{C}$, and the free-energy cutoff was set to $-22 \mathrm{kcal} / \mathrm{mol}$. Of the primary candidate miRNA sequences, those miRNAs that were confirmed by another program as rna22 (35) were selected as candidates. miRanda was run according to the authors' recommendation.

Reporter plasmids and reporter assay. The vector pSPORT1-mTIMP1, which contains full open reading frame sequences of mTIMP1 (IMAGE ID: 30056565), was purchased from ATCC. The reporter plasmid was constructed by subcloning PCR-amplified 3'-UTR fragments from the pSPORT-mTIMP1 into the downstream of a Photinus pyralis (firefly) luciferase reporter gene using EcoR1 and Kpn1 sites. To check the transfection efficiency, pRL-TK, a control plasmid (Promega), was used. Transfection was performed using Lipofectamine 2000 (Invitrogen). Luciferase assays were carried out with the Dual-Luciferase Reporter Assay System (Promega).

Expression plasmids. pcDNA3-TIMP1 expression vector was constructed by subcloning PCR-amplified coding sequence fragments from the PSPORTmTIMP1 with EcoR1 and Kpn1 sites. pSuper-miR378, pSuper-miR17-5p, and pSuper-let7b, which express miR378, miR17-5p, and let7b, respectively, were constructed by cloning the miR sequences into the pSuper vector using XhoI and BglII sites.

Western blotting analysis and antibodies. The cell extracts and homogenized mouse tissues were normalized for protein concentration using the BioRad Dc Protein Assay Kit (Bio-Rad), or, for the detection of TIMP1 in the overexpression of miRNA, cell extracts were normalized based on the luciferase value of cotransfected pRL-TK to avoid the variances of transfection efficiency. Protein $(30 \mu \mathrm{g})$ was resolved by SDS-PAGE, transferred to PVDF (Hybond-P; Amersham Pharmacia Biotech), and immunoblotted. A goat polyclonal antibody against mTIMP1 was purchased from R\&D systems. A rabbit monoclonal anti-VEGF receptor 2 antibody (55B11) was purchased from Cell Signaling. A mouse monoclonal anti-VEGF receptor 1 (Flt-1) antibody (MAB1664) and a mouse anti-GAPDH antibody were purchased from Chemicon. The bound antigens were detected using SuperSignal West Femto Maximum Sensitivity Substrate (Pierce).

Cells. The mouse endothelial cell line SVEC4-10 and the human embryonic kidney cell line 293T were obtained from ATCC and cultured in DMEM supplemented with $10 \%$ FBS.

Reverse zymography. SVEC or 293T cell culture supernatant after transfection of the TIMP1 expression vector with miRNA expression plasmids anti-miRNA oligos, or miRNA precursors were analyzed for the activity of TIMP1 and MMP2 as described previously (46). Because MMPs are separated from their inhibitor TIMP1 during the normal zymographic procedure, their activities cannot be analyzed accurately. To avoid this problem and better define the balance existing between MMP2 and its inhibitor TIMP1, we measured the TIMP1 activity by reverse zymography. Briefly, 36 hours after transfection (control antisense oligos with random sequences, anti-miR17-5p and let7b oligonucleotides, control miRNA precursors with random sequences, or double-stranded miR $17-5 \mathrm{p}$ and let $7 \mathrm{~b}$ precursors), supernatants were collected, concentrated 10-fold using the Centricon concentrator (Amicon), and electrophoresed on nondenaturing $0.1 \%$ 
SDS, $12 \%$ polyacrylamide gels containing $1 \mathrm{mg} / \mathrm{ml}$ gelatin, and $0.16 \mu \mathrm{g} / \mathrm{ml}$ 4-aminophenylmercuric acetate-activated MMP-2 (both from EMD). Electrophoresis was carried out at $4^{\circ} \mathrm{C}$, and then the gel was renatured in $2.5 \%$ Triton X-100 for 3 hours with 3 times replacement of fresh solution at room temperature. Then, the gel was incubated in enzyme buffer $(50 \mathrm{mM}$ Tris, pH 7.5, $200 \mathrm{mM} \mathrm{NaCl}, 5 \mathrm{mM} \mathrm{CaCl}$, and $0.02 \%$ Brij-35) for 24 hours at $37^{\circ} \mathrm{C}$ and stained with Coomassie blue G-250. The gel image and the integrated intensities were obtained and quantified using FluorChem8900.

Endothelial tube formation assay, cell proliferation assay, and migration assay. AntimiRNA17-5p and let7b oligonucleotides, double-stranded miRNA17-5p, and let7b precursors and random-sequence anti-miRNA oligos and miRNA precursors were purchased from Ambion; $40 \mathrm{nM}$ anti-miRNA oligos or $40 \mathrm{nM}$ miRNA precursors were transfected into SVEC. Control antisense oligos with random sequences and control miRNA precursors with random sequences were used as negative controls. Thirty-six hours after transfection, $1 \times 10^{5}$ cells were seeded in a 12-well plate coated with $200 \mu \mathrm{l}$ Growth Factor Reduced Matrigel (BD Biosciences). Tube length was quantified after 12 and 18 hours by measuring the cumulative tube length in 3 random microscopic fields with a computer-assisted microscope using the program AxioVision 3.1 (Carl Zeiss). For the cell proliferation assay, 36 hours after transfection, $5 \times 10^{4}$ cells were seeded and the number of cells was determined by cell counting at the indicated time points. For the examination of cell motility, an in vitro wound healing assay was performed. Transfected cells were seeded with $2.5 \times 10^{6}$ cells in a 12 -well plate and cultured in 1\% FBS for 24 hours to synchronize and were then wounded by removing a 200- to $400-\mathrm{mm}$ strip of cells across the well with a $200-\mu l$ pipette tip. Wound healing was quantified as the average length of the elongation of wound edges over 24 hours by using AxioVision 3.1.

Progesterone assay. Serum progesterone levels were measured in serum samples collected at indicated days after coitus using a rodent progesterone ELISA kit according to the manufacturer's instruction (Endocrine Technologies Inc.). The limit of sensitivity of the assay was $0.1 \mathrm{ng} / \mathrm{ml}$.

Semiquantitative RT-PCR analysis. Total RNA was extracted from ovaries using Trizol reagent (Invitrogen), and RT-PCR was done as described previously (13). The primers used were as follows: cytochrome P450 family 11 subfamily a polypeptide 1 , 5'-AGAAGCTGGGCAACATGGAGTCAG-3' and $5^{\prime}$-TCACATCCCAGGCAGCTGCATGGT-3'; luteinizing hormone receptor, 5'-CTTATACATAACCACCATACCAG-3' and 5'-ATCCCAGCCACTGAGTTCATTC- $3^{\prime}$; prolactin receptor, $5^{\prime}$-ATACTGGAGTAGATGGGGCCAGGAGAAATC- $3^{\prime}$ and $5^{\prime}$-CTTCCATGACCAGAGTCACTGTCAGGATCT- $3^{\prime}$. The result was quantified using FluorChem software and normalized by GAPDH intensities.

miRNA injection technique. Mice were anesthetized using ketamine. A dorsolateral small incision was made in the lumber region on each side of the midline. Through the peritoneal incision, the ovaries were gently pulled outside the body. The intrabursal injection was performed under microscopic magnification by inserting a 30 -gauge needle through the ovarian fat pad into the ovarian bursa. The ovaries were returned to the abdominal cavity, and the wound was sutured with 4-0 silk (Ethicon Inc.). For histological analyses, 5 female Dicer ${ }^{d / d}$ mice were used. In each mouse, a 50- $\mu 1$ mixture of $0.5 \mathrm{nmol}$ double-stranded miRNA17-5p and let $7 \mathrm{~b}$ precursors with normal saline and lipofectamine 2000 was delivered into the right ovary, and transfection reagents alone were injected into the left ovary as a control. To examine the course of pregnancy, 5 Dicerd/d female mice were injected with miRNAs into both ovaries. To determine the injection and transfection efficiency, Cy-3-labeled, fluorescent siRNA (siGLO; Dharmacon) was injected in the same way. Injection of transfection reagent alone was used as control. Three days after the procedure, the ovaries were sectioned, frozen, and examined under a fluorescent microscope. Mice were initially housed separately; when they resumed normal behavioral activity, they were housed with a fertile male for mating. Vaginal plugs were confirmed the next morning (day 0.5 of pregnancy). For the histological analyses, mice were sacrificed on day 1.5 , and the ovaries were sectioned, frozen, and then examined histologically. Western blotting of TIMP1 and the antibody analyses were performed using the lysates of control, and the injected ovaries were extracted on day 1.5 of pregnancy. For the analyses of serum progesterone levels, serum samples were collected on the indicated days of pregnancy from 4 Dicer ${ }^{d / d}$ mice in which both ovaries had been injected. In the case of $\mathrm{Dicer}^{+/+}$mice, the same methods were used, except that anti-miRNA17-5p and let7b oligos were used instead of miRNA precursors.

Statistics. The statistical significance of any differences was determined using the 2-tailed Student's $t$ test or Welch's $t$ test when variances were unequal.

\section{Acknowledgments}

We thank Kaoru Saigo for providing the Dicer antibodies and Ryan Cook for critically reading the manuscript. This work was supported by NIH grants GM67101, AI41637, and AI54696 and by 863 program grant AA02Z164. M. Otsuka was supported by the Sankyo Foundation of Life Science Fellowship and the Uehara Memorial Foundation Research Fellowship.

Received for publication August 21, 2007, and accepted in revised form February 6, 2008.

Address correspondence to: Jiahuai Han, Department of Immunology, IMM-32, The Scripps Research Institute, 10550 North Torrey Pines Road, La Jolla, California 92037, USA. Phone: (858) 784-8704; Fax: (858) 784-8665; E-mail: jhan@scripps.edu.

Masaaki Hayashi's present address is: Lexicon Pharmaceuticals, The Woodlands, Texas, USA.
1. Hannon, G.J. 2002. RNA interference. Nature. 418:244-251.

2. Carmell, M.A., and Hannon, G.J. 2004. RNase III enzymes and the initiation of gene silencing. Nat. Struct. Mol. Biol. 11:214-218.

3. Bartel, D.P. 2004. MicroRNAs: genomics, biogenesis, mechanism, and function. Cell. 116:281-297.

4. Meister, G., and Tuschl, T. 2004. Mechanisms of gene silencing by double-stranded RNA. Nature. 431:343-349.

5. Jing, Q., et al. 2005. Involvement of microRNA in AU-rich element-mediated mRNA instability. Cell. 120:623-634.

6. Bernstein, E., et al. 2003. Dicer is essential for mouse development. Nat. Genet. 35:215-217.

7. Yang, W.J., et al. 2005. Dicer is required for embryonic angiogenesis during mouse development.
J. Biol. Chem. 280:9330-9335.

8. Harfe, B.D., McManus, M.T., Mansfield, J.H., Hornstein, E., and Tabin, C.J. 2005. The RNaseIII enzyme Dicer is required for morphogenesis but not patterning of the vertebrate limb. Proc. Natl. Acad. Sci. U. S. A. 102:10898-10903.

9. Muljo, S.A., et al. 2005. Aberrant T cell differentiation in the absence of Dicer. J. Exp. Med. 202:261-269.

10. Cobb, B.S., et al. 2005. T cell lineage choice and differentiation in the absence of the RNase III enzyme Dicer. J. Exp. Med. 201:1367-1373.

11. Yi, R., et al. 2006. Morphogenesis in skin is governed by discrete sets of differentially expressed microRNAs. Nat. Genet. 38:356-362.

12. Harris, K.S., Zhang, Z., McManus, M.T., Harfe, B.D., and Sun, X. 2006. Dicer function is essential for lung epithelium morphogenesis. Proc. Natl. Acad. Sci. U. S. A. 103:2208-2213.

13. Otsuka, M., et al. 2007. Hypersusceptibility to vesicular stomatitis virus infection in Dicer1-deficient mice is due to impaired miR24 and miR93 expression. Immunity. 27:123-134.

14. Risau, W. 1997. Mechanisms of angiogenesis. Nature. 386:671-674.

15. Adams, R.H., and Alitalo, K. 2007. Molecular regulation of angiogenesis and lymphangiogenesis. Nat. Rev. Mol. Cell Biol. 8:464-478.

16. Poliseno, L., et al. 2006. MicroRNAs modulate the angiogenic properties of HUVECs. Blood. 108:3068-3071.

17. Dews, M., et al. 2006. Augmentation of tumor angiogenesis by a Myc-activated microRNA cluster. Nat. Genet. 38:1060-1065. 
18. Kuehbacher, A., Urbich, C., Zeiher, A.M., and Dimmeler, S. 2007. Role of Dicer and Drosha for endothelial microRNA expression and angiogenesis. Circ. Res. 101:59-68.

19. Suarez, Y., Fernandez-Hernando, C., Pober, J.S., and Sessa, W.C. 2007. Dicer dependent microRNAs regulate gene expression and functions in human endothelial cells. Circ. Res. 100:1164-1173.

20. Reynolds, L.P., Grazul-Bilska, A.T., and Redmer, D.A. 2000. Angiogenesis in the corpus luteum. Endocrine. 12:1-9.

21. Reynolds, L.P., Killilea, S.D., and Redmer, D.A. 1992. Angiogenesis in the female reproductive system. FASEB J. 6:886-892.

22. Ferrara, N., et al. 1998. Vascular endothelial growth factor is essential for corpus luteum angiogenesis. Nat. Med. 4:336-340.

23. Nardo, L.G., and Sallam, H.N. 2006. Progesterone supplementation to prevent recurrent miscarriage and to reduce implantation failure in assisted reproduction cycles. Reprod. Biomed. Online. 13:47-57.

24. Jones, G.E. 1949. Some newer aspects of the management of infertility. J. Am. Med. Assoc. 141:1123-1129.

25. Hinney, B., Henze, C., Kuhn, W., and Wuttke, W. 1996. The corpus luteum insufficiency: a multifactorial disease. J. Clin. Endocrinol. Metab. 81:565-570.

26. Pritts, E.A., and Atwood, A.K. 2002. Luteal phase support in infertility treatment: a meta-analysis of the randomized trials. Hum. Reprod. 17:2287-2299.

27. Murchison, E.P., et al. 2007. Critical roles for Dicer in the female germline. Genes Dev. 21:682-693.

28. Tang, F., et al. 2007. Maternal microRNAs are essential for mouse zygotic development. Genes Dev. 21:644-648.
29. Hsieh, M., et al. 2005. Mice null for Frizzled4 (Fzd4-/-) are infertile and exhibit impaired corpora lutea formation and function. Biol. Reprod. 73:1135-1146.

30. Grosdemouge, I., et al. 2003. Effects of deletion of the prolactin receptor on ovarian gene expression. Reprod. Biol. Endocrinol. 1:12.

31. Xu, Q., et al. 2004. Vascular development in the retina and inner ear: control by Norrin and Frizzled-4, a high-affinity ligand-receptor pair. Cell. 116:883-895.

32. Liu, K., Wahlberg, P., Hagglund, A.C., and Ny, T. 2003. Expression pattern and functional studies of matrix degrading proteases and their inhibitors in the mouse corpus luteum. Mol. Cell. Endocrinol. 205:131-140.

33. Reynolds, L.P., Grazul-Bilska, A.T., and Redmer, D.A. 2002. Angiogenesis in the female reproductive organs: pathological implications. Int. J. Exp. Pathol. 83:151-163.

34. Rusinov, V., Baev, V., Minkov, I.N., and Tabler, M. 2005. MicroInspector: a web tool for detection of miRNA binding sites in an RNA sequence. Nucleic Acids Res. 33:W696-W700.

35. Miranda, K.C., et al. 2006. A pattern-based method for the identification of MicroRNA binding sites and their corresponding heteroduplexes. Cell. 126:1203-1217.

36. John, B., et al. 2004. Human MicroRNA targets. PLoS Biol. 2:e363

37. Lewis, B.P., Shih, I.H., Jones-Rhoades, M.W., Bartel, D.P., and Burge, C.B. 2003. Prediction of mammalian microRNA targets. Cell. 115:787-798.

38. Akahane, T., et al. 2004. TIMP-1 inhibits microvas- cular endothelial cell migration by MMP-dependent and MMP-independent mechanisms. Exp. Cell Res. 301:158-167.

39. Handsley, M.M., and Edwards, D.R. 2005. Metalloproteinases and their inhibitors in tumor angiogenesis. Int. J. Cancer. 115:849-860.

40. Khokha, R.,Zimmer, M.J., Graham, C.H., Lala, P.K. and Waterhouse, P. 1992. Suppression of invasion by inducible expression of tissue inhibitor of metalloproteinase-1 (TIMP-1) in B16-F10 melanoma cells. J. Natl. Cancer Inst. 84:1017-1022.

41. Madri, J.A., Pratt, B.M., and Tucker, A.M. 1988. Phenotypic modulation of endothelial cells by transforming growth factor-beta depends upon the composition and organization of the extracellular matrix. J. Cell Biol. 106:1375-1384.

42. Matzuk, M.M., and Lamb, D.J. 2002. Genetic dissection of mammalian fertility pathways. Nat. Cell Biol. 4:s41-s49.

43. Wang, H., and Dey, S.K. 2006. Roadmap to embryo implantation: clues from mouse models. Nat. Rev. Genet. 7:185-199.

44. Roy, A., and Matzuk, M.M. 2006. Deconstructing mammalian reproduction: using knockouts to define fertility pathways. Reproduction. 131:207-219.

45. Inzunza, J., et al. 2007. Ovarian wedge resection restores fertility in estrogen receptor beta knockout (ERbeta-/-) mice. Proc. Natl. Acad. Sci. U. S. A. 104:600-605

46. Oliver, G.W., Leferson, J.D., Stetler-Stevenson, W.G., and Kleiner, D.E. 1997. Quantitative reverse zymography: analysis of picogram amounts of metalloproteinase inhibitors using gelatinase A and B reverse zymograms. Anal. Biochem. 244:161-166. 